\title{
DECOMPOSITIONS OF COMPLETE GRAPHS INTO KAYAK PADDLES
}

\author{
Dalibor Froncek ${ }^{1}$, Leah TOllefson ${ }^{2}$ \\ ${ }^{1}$ Department of Mathematics and Statistics, University of Minnesota \\ Duluth, 1117 University Drive, Duluth, MN 55812-3000, U.S.A. \\ ${ }^{2}$ Department of Mathematics and Statistics, University of Minnesota \\ Duluth, 1117 University Drive, Duluth, MN 55812-3000, U.S.A.
}

\begin{abstract}
A canoe paddle is a cycle attached to an end-vertex of a path. It was shown by Truszczyński that all canoe paddles are graceful and therefore decompose complete graphs. A kayak paddle is a pair of cycles joined by a path. We prove that the complete graph $K_{2 n+1}$ is decomposable into kayak paddles with $n$ edges whenever at least one of its cycles is even.

Key words: Graph decomposition, graceful labeling, rosy labeling.
\end{abstract}

2000 Mathematics Subject Classification: 05C78.

Received: 09-08-2011, revised: 09-09-2011, accepted: 04-12-2012. 